\title{
Quantum Phase Transition in the Two-Band Hubbard Model
}

\author{
T. A. Costi and A. Liebsch \\ Institut für Festkörperforschung, Forschungszentrum Jülich, 52425 Jülich, Germany
}

(Received 25 July 2007; published 7 December 2007)

\begin{abstract}
The interaction between itinerant and Mott localized electronic states in strongly correlated materials is studied within dynamical mean field theory in combination with the numerical renormalization group method. A novel nonmagnetic zero temperature quantum phase transition is found in the bad-metallic orbital-selective Mott phase of the two-band Hubbard model, for values of the Hund's exchange which are relevant to typical transition metal oxides.
\end{abstract}

DOI: 10.1103/PhysRevLett.99.236404

PACS numbers: 71.27.+a, 71.10.- w, 71.30.+h, 72.15.Qm

The nature of the metal insulator transition in multicomponent systems, with competing energy scales associated with differing bandwidths and Coulomb and exchange interactions, is currently of great interest [1]. For instance, in transition metal oxides such as $\mathrm{Ca}_{2-x} \mathrm{Sr}_{x} \mathrm{RuO}_{4}$ or manganites (e.g., $\mathrm{La}_{1-x} \mathrm{Sr}_{x} \mathrm{MnO}_{3}$ ), the Coulomb interaction between weakly and strongly correlated subbands can give rise to complex phase changes as a function of temperature, pressure, or impurity concentration. An interesting aspect of such systems is the coexistence of itinerant wide band electrons with partially or completely localized narrow band electrons. This gives rise to bad-metallic behavior as observed, for example, in the resistivity of the paramagnetic phases of $\mathrm{VO}_{2}$ [2] and $\mathrm{Ca}_{2-x} \mathrm{Sr}_{x} \mathrm{RuO}_{4}$ at $x=0.2$ [3]. Even in standard Mott insulators such as $\mathrm{V}_{2} \mathrm{O}_{3}$ and $\mathrm{LaTiO}_{3}$, the presence of inequivalent orbitals leads to strong orbital polarization where in the vicinity of the transition nearly localized electrons coexist with weakly itinerant bands [4-6]. In the case of cuprates, one of the most fascinating aspects is the coexistence of strongly and weakly correlated regions of the Brillouin zone, giving rise to so-called hot spots and cold spots, with breakdown of Fermi-liquid behavior in the former and Fermi-liquid properties in the latter [7-9]. Since the momentum variation of the self-energy is associated with spatial fluctuations, an intriguing analogy exists between multisite interactions within a single band and single-site interactions among inequivalent orbitals.

A simple model which captures some of the essential physics occurring in the systems mentioned above is the two-band Hubbard model consisting of narrow and wide subbands, coupled via local Coulomb energy $U$ and Hund's exchange $J[10,11]$. As a result of the various energy scales contained in this model, its phase diagram turns out to be remarkably rich, as shown in detailed studies by many authors [12-21]. These studies revealed that differing bandwidths and a finite Hund's exchange stabilize an orbital-selective Mott phase, in which the wide band itinerant electrons coexist with localized spins arising from Mott localization of the narrow band electrons. Further increase of the Coulomb energy $U$ leads to a Mott tran- sition in the wide band whose nature has remained unresolved for the important case of anisotropic Hund's exchange of relevance to many transition metal compounds.

The aim of the present work is to identify the character of the wide band transition of the two-band Hubbard model at zero temperature for anisotropic Hund's exchange. We use dynamical mean field theory (DMFT) [22] in combination with the numerical renormalization group (NRG) method [23-26] in order to achieve a sufficiently accurate description of low-frequency properties. We find that anisotropic Hund's exchange yields a continuous quantum phase transition as long as $J / U>j_{c}$, where $j_{c} \approx 0.1$. This range of $J / U$ values is typical of transition metal oxides (e.g., for manganites). In this region, the spectral density $A(\omega)$ vanishes at the Fermi level as $|\omega|^{\delta}$, with $\delta=1 / 3$. This behavior coincides with the one for the metal insulator transition in the exactly solvable limit $U=0$, with variable $J$. In striking contrast, the region $0<J / U<j_{c}$ exhibits a first-order Mott transition, which differs from the continuous zero temperature Mott transition for the one-band model [22].

We consider an effective low-energy model specific for the orbital-selective Mott phase [21] which allows studying the transition of the wide band in detail. In this model, the electrons in the narrow band of the original two-band Hubbard model have undergone Mott localization so their low-energy degrees of freedom are represented by localized spins. These couple to the itinerant wide band electrons via a Hund's exchange. The resulting model is the ferromagnetic Kondo lattice model with interactions in the band,

$$
\begin{aligned}
H_{\mathrm{fkl}}= & -\sum_{i j \sigma} t_{i j} c_{i \sigma}^{\dagger} c_{j \sigma}+U \sum_{i} n_{i \uparrow} n_{i \downarrow} \\
& -\sum_{i}\left[2 J S_{i}^{z} s_{i}^{z}+2 J^{\prime}\left(S_{i}^{+} s_{i}^{-}+S_{i}^{-} s_{i}^{+}\right)\right],
\end{aligned}
$$

where $s_{i}^{z}$ and $s_{i}^{ \pm}$are the $z$ component and raising or lowering operators, respectively, for electrons in the wide band at site $i$, and $S_{i}^{z}$ and $S_{i}^{ \pm}$are the corresponding operators for the localized spin at site $i$. The anisotropy of the Hund's exchange is measured by $J^{\prime} / J \leq 1$ with $J^{\prime}=J$ corre- 
sponding to isotropic and $J^{\prime}=0$ to Ising Hund's exchange. The effective impurity model corresponding to (1) which we solve within DMFT-NRG is the ferromagnetic Kondo model with a local Coulomb repulsion. As in previous works we used a Bethe-lattice density of states with half bandwidth $D=1$, which we henceforth take as our unit of energy. In the NRG procedure 800-1200 states per energy shell were retained. The logarithmic discretization parameter for the conduction band was $\Lambda=1.5$ and $N=90$ shells were used, corresponding to a minimum energy resolution of $\Lambda^{-(N-1) / 2} \approx 10^{-8}$. The calculations have been carried out for fixed values of $J / U$ and increasing values of $U / D$, as we consider the situation where correlations are tuned by reducing the bandwidth $D$ whereas the ratio $J / U$ is material dependent, being, for example, larger than unity for manganites and smaller than unity for most transition metal oxides. As we are interested in the metal insulator transition, we consider a half-filled band and paramagnetic solutions only.

Note that the model (1) can also be viewed as a singleband Hubbard model in the presence of spin-disorder scattering of strength given by the Hund's exchange. For $U=0$ and $J>0$ the model reduces to the double exchange model used to describe manganites which is known to exhibit non-Fermi-liquid behavior on restricting to paramagnetic ground states [27]. This non-Fermi-liquid or badmetallic behavior persists to $U>0[11,21,28]$.

In the present work we focus on the Ising limit, i.e., $J^{\prime}=$ 0 . While this might appear as a severe restriction, this limit is actually relevant for all cases with $J^{\prime}<J$, and therefore should be important in materials where as a result of noncubic octahedral distortions Hund's exchange will not be fully isotropic. This can be understood from the wellknown renormalization group flow of the ferromagnetic Kondo impurity model [29] and explicit numerical calculations including the local Coulomb interaction $U$ [30]. For a metallic band, these predict that for anisotropic Hund's exchange, $J^{\prime}<J$, the spin-flip part of the exchange, $J^{\prime}$, renormalizes to zero, but the Ising part, $J$, renormalizes to a finite value $J^{*}>0$. Similarly, for an insulating band, one finds that the Ising coupling renormalizes to a finite value and the system is gapped so $J^{\prime}<J$ becomes irrelevant for low-energy properties. From these arguments it follows that, for the purposes of determining the nature of the Mott transition in the wide band for anisotropic Hund's exchange, it suffices to consider just Ising anisotropy $J^{\prime}=$ 0 . Results for general $J^{\prime}$ will be given elsewhere [30]. A finite Ising exchange for metallic and insulating cases also implies that the entropy of the lattice model (1) is $\ln 2$ per site for both metallic and insulating states. Consequently, the metal insulator transition at finite temperature need not be first order, and, in particular, a continuous quantum phase transition can arise at $T=0$ which becomes a crossover at finite temperature. We also note that the renormalization of $J^{\prime}$ to $J^{*}=0$ in the metallic case implies that the spin disorder in the lattice model (1) persists to $T=0$, giving rise to above-mentioned bad-metal behavior with $\operatorname{Im}[\Sigma(\omega=0)]<0[11,21,28]$, where $\Sigma$ is the wide band conduction electron self-energy.

To provide an overview of the different transition regimes of the model defined in (1) we show first in Fig. 1 the behavior of the effective conduction electron magnetic moment, $\left\langle s_{z}^{2}\right\rangle$, as a function of increasing or decreasing $U$ for several fixed values of $J / U$. For $J / U=0$ we recover the Mott transition in the single-band Hubbard model with a hysteresis region and a continuous metal insulator transition at $U=U_{c 2} \approx 3$. The hysteresis behavior persists for small but finite exchange although it rapidly diminishes with increasing $J / U$ (see inset to Fig. 1). Within the numerical accuracy of our calculations, the hysteresis behavior vanishes for $J / U>j_{c} \approx 0.1$, indicating that the Mott transition in this region is a continuous quantum phase transition. The critical Coulomb interaction for the Mott transition is seen to decrease monotonically with increasing $J / U$ (inset to Fig. 1). The precise location and nature of the critical point at $J / U=j_{c} \approx 0.1, U=u_{c} \approx$ 1.6 where first and second order lines meet requires a detailed study and is outside the scope of the present Letter. The phase diagram shown in Fig. 1 is consistent with ED-DMFT results at low $T$ which also yield firstorder behavior for $J / U<0.1, U<u_{c} \approx 1.6$ and a continuous transition for $J / U>0.1, U>u_{c}$ [30].

Let us now look closer at the continuous quantum phase transition for $J / U>j_{c}$. Figure 2(a) shows the evolution of

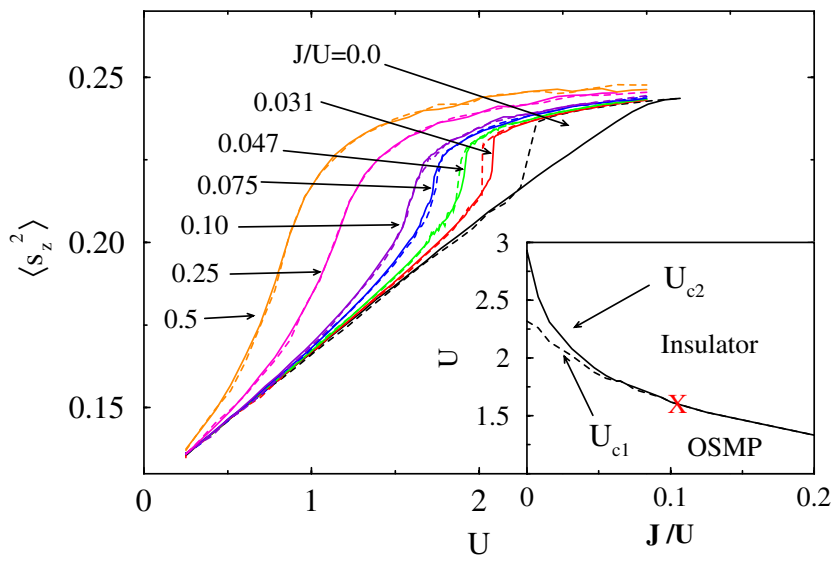

FIG. 1 (color online). Effective magnetic moment $\left\langle s_{z}^{2}\right\rangle$ per site of the conduction electrons for several values of $J / U, J^{\prime}=0$ as a function of $U$. Solid (dashed) lines: increasing (decreasing) values of $U$. For $0<J / U<0.1$ the Mott transition is of first order with hysteresis in a region $U_{c 1}<U<U_{c 2}$ (the lines are guides to the eye; a finer grid of $U$ points, as used for $J / U=$ 0.031 , reveals vertical jumps at $\left.U_{c 1,2}\right)$. For $J / U>0.1$ the transition becomes a continuous quantum phase transition. Inset: $T=0$ phase diagram and dependence of $U_{c 1,2}$ on $J / U$. OSMP denotes the orbital-selective Mott phase. The red $\times$ denotes the critical point $J / U=j_{c} \approx 0.1, U=u_{c} \approx 1.6$, where the Mott transition changes from first to second order. 

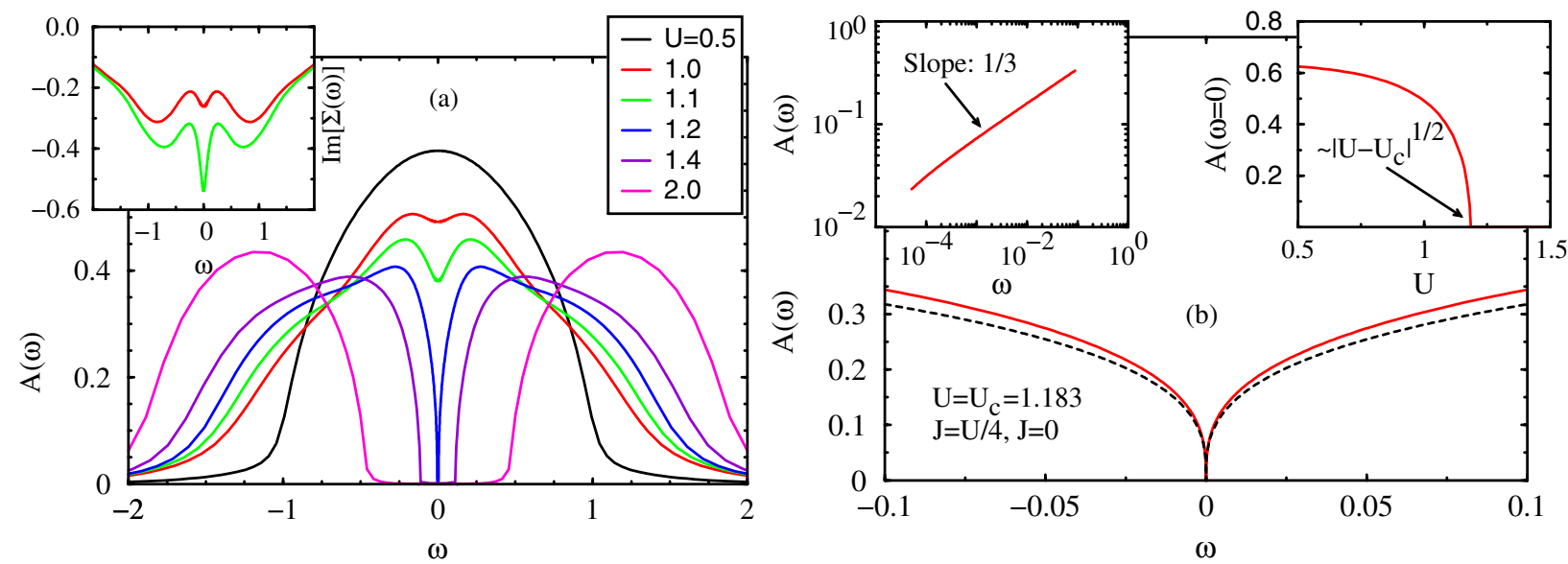

FIG. 2 (color online). Left panel: spectral density of wide band for several values of $U$, for $J=U / 4, J^{\prime}=0$. The Mott transition occurs at $U_{c}=1.183$ and is continuous. Inset: imaginary part of the self-energy for $U<U_{c}$, indicating a bad metal. Right panel: lowfrequency region of spectral density $A(\omega)$ for $J=U / 4, J^{\prime}=0$ at $U_{c}=1.183$ (red solid line; logarithmic plot left inset); the analogous spectrum for the exactly solvable model at $J_{c}=2, U=0$, is shown by the dashed line. Right inset: $A(0)$ as a function of $U$.

the spectral density as a function of $U$ for fixed $J / U=$ $1 / 4$. For $J^{\prime} \approx J$, this would be a value typical for transition metal oxides which has been used in many previous calculations $[11-18,20]$. At small $U$, the density of states at the Fermi level, $E_{F}=0$, satisfies the pinning condition, $A(0)=2 / \pi$, approximately. Near $U \approx 1$ the spectrum begins to exhibit a pseudogap which gets progressively deeper with increasing $U$. The characteristic scale for this pseudogap is $J$. At $U_{c}=1.183$ this pseudogap becomes a soft gap before a full insulating gap opens at $U>$ $U_{c}$. This critical value is in reasonable agreement with EDDMFT results for $T=0$ [13] and $T>0$ [12]. In the badmetallic region below the Mott transition, the electronic lifetime $\tau(\omega=0) \sim-1 / \operatorname{Im}[\Sigma(\omega=0)]$ is finite [see inset to Fig. 2(a)] and vanishes at $U=U_{c}$. Note that for this relatively large value of $J / U$, well-defined upper and lower Hubbard bands are only formed once the insulating gap has opened. In contrast, for smaller values of $J / U$ well-defined Hubbard bands are formed already in the metallic state, see Fig. 3 below. These $T=0$ spectra are consistent with those derived within quantum Monte Carlo (QMC) DMFT at finite $T[11,20,31]$. Because of the presence of the pseudogap the spectra exhibit a characteristic four-peaked structure, with low-energy features limiting the gap and high-energy peaks associated with Hubbard bands.

We have analyzed the low-frequency behavior of the spectral density at the quantum phase transition in the region $J / U>j_{c}$ and find $A(\omega) \sim|\omega|^{\delta}$ with $\delta=1 / 3$, as shown in Fig. 2(b). Moreover, $A(0) \sim\left|U-U_{c}\right|^{1 / 2}$ for $U \rightarrow U_{c}^{-}$. Remarkably, this behavior coincides with the one obtained for the continuous Mott transition in the exactly solvable model $J / U \rightarrow \infty$ with $U \rightarrow 0$ on increasing $J$. Solving the DMFT equations for this model, we find a band-splitting transition at $J_{c}=2$ with $A(\omega) \sim|\omega|^{1 / 3}$ and $A(0) \sim\left|J-J_{c}\right|^{1 / 2}$ for $J \rightarrow J_{c}^{-}$[32]. This suggests that the low-frequency excitations in the whole region $J / U>$ $j_{c}$ are governed by the Ising Hund's exchange and that the paramagnetic metal insulator transition belongs to the same universality class as the band-splitting transition in the model with $U=0$ on increasing $J$.

We now discuss the region $J / U<j_{c}$ where the effective two-band model (1) yields a first-order Mott transition at $T=0$. This is evidenced by the discontinuity in $\left\langle s_{z}^{2}\right\rangle$ at both $U_{c 1}$ and $U_{c 2}$ (see Fig. 1) and in the discontinuous jump in the spectral density at $E_{F}$ (see inset to Fig. 3). A comparison of the ground state energies of metallic and insulating solutions in the hysteresis regions shows that the metallic solution is lower in energy than the insulating one, so the first-order transition at finite $J / U$ occurs at $U_{c 2}$ as for the $T=0$ metal insulator transition in the single-band model. The spectral densities for $J / U=0.031$ shown in

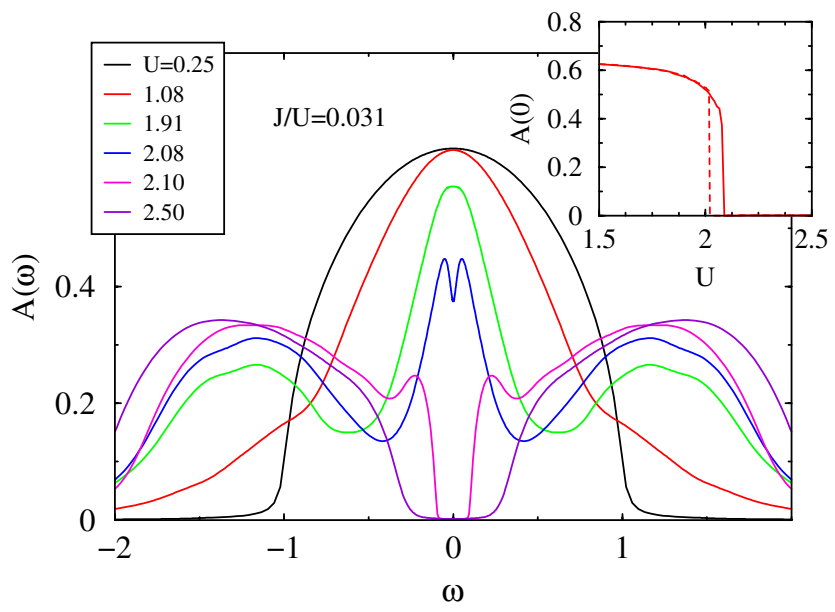

FIG. 3 (color online). Spectral densities of the wide band for several values of $U$ for $J / U=0.031, J^{\prime}=0$. The Mott transition is first order and occurs at $U_{c} \approx 2.09$. Inset: $A(0)$ as a function of $U$ showing hysteresis and discontinuous jump. Solid (dashed) lines: increasing (decreasing) values of $U$. 
Fig. 3 demonstrate that, as for $J / U=1 / 4$, the pinning condition $A(0)=2 / \pi$ is increasingly violated with increasing $U$, indicating the bad-metallic nature of the phase below the transition. Because of the smaller value of $J$ the pseudogap is considerably narrower than for $J / U=1 / 4$. As a result, the characteristic four-peaked structure in the bad-metallic phase is now better resolved than for the ones presented in Fig. 2(a). Note that, despite the small value of $J$, the spectra shown in Fig. 3 differ markedly from the corresponding spectra of the single-band Hubbard model obtained in the limit $J=0$. Ising Hund's exchange leads to a significant redistribution of spectral weight at low frequencies so that even a weak coupling to the localized spins creates a pseudogap and destroys the zero-frequency pinning condition. We find that these effects persist also for weak anisotropies $J^{\prime} / J \approx 0.8-0.9$ [30].

In summary, we have studied the metal insulator transition in the two-band Hubbard model with bands of different widths and for Ising Hund's exchange, arguing that the results for the metal insulator transition in the wide band apply for all anisotropies $J^{\prime}<J$. The present NRG-DMFT calculations allow a sufficiently accurate analysis of the low-frequency properties of the wide band in the case of anisotropic Hund's exchange. The results reveal a continuous quantum phase transition for $J / U>0.1$, i.e., in the region relevant for many transition metal oxide materials. Close inspection of the critical properties of this transition show that they coincide with the ones of the band-splitting transition in the model with $U=0$ and variable $J$. In the region $J / U<0.1$, the transition becomes first order with hysteresis. This can be interpreted as the stability of firstorder transitions, in this case that at $J=0$ for small finite temperatures, to weak perturbations such as the weak coupling to localized spins. A sufficiently large perturbation destroys this first-order behavior, giving rise, in the present case, to a continuous quantum phase transition for $J / U>0.1$. The wide band is shown to be bad metallic for all $J>0$, with a low-frequency pseudogap whose width is determined by $J$ and Hubbard bands whose positions are determined by $U$. The resulting four-peaked spectral densities differ fundamentally from the familiar three-peaked structures of the one-band model to which our model reduces in the limit $J=0$ [33]. We have also argued that the present results for fully anisotropic Hund's exchange $\left(J^{\prime}=0\right)$ are relevant to the generic case of weakly anisotropic exchange $\left(J^{\prime}<J\right)$ with the critical properties of the wide band transition remaining unaltered for $J^{\prime}<J$.

We would like to thank A. Rosch for valuable discussions. Supercomputing support from the John von Neumann Institute (Jülich) is gratefully acknowledged.

[1] M. Imada, A. Fujimori, and Y. Tokura, Rev. Mod. Phys. 70, 1039 (1998).
[2] P. B. Allen et al., Phys. Rev. B 48, 4359 (1993).

[3] S. Nakatsuji and Y. Maeno, Phys. Rev. B 62, 6458 (2000).

[4] G. Keller et al., Phys. Rev. B 70, 205116 (2004).

[5] A. I. Poteryaev et al., Phys. Rev. B 76, 085127 (2007).

[6] E. Pavarini et al., Phys. Rev. Lett. 92, 176403 (2004).

[7] M. Civelli et al., Phys. Rev. Lett. 95, 106402 (2005).

[8] B. Kyung et al., Phys. Rev. B 73, 165114 (2006).

[9] Y.Z. Zhang and M. Imada, Phys. Rev. B 76, 045108 (2007).

[10] V. I. Anisimov et al., Eur. Phys. J. B 25, 191 (2002).

[11] A. Liebsch, Phys. Rev. B 70, 165103 (2004); Phys. Rev. Lett. 91, 226401 (2003).

[12] A. Liebsch, Phys. Rev. Lett. 95, 116402 (2005).

[13] A. Koga et al., Phys. Rev. Lett. 92, 216402 (2004).

[14] M. Ferrero et al., Phys. Rev. B 72, 205126 (2005).

[15] L. de'Medici, A. Georges, and S. Biermann, Phys. Rev. B 72, 205124 (2005).

[16] A. Rüegg et al., Eur. Phys. J. B 48, 55 (2005).

[17] R. Arita and K. Held, Phys. Rev. B 72, 201102(R) (2005).

[18] K. Inaba and A. Koga, Phys. Rev. B 73, 155106 (2006); for details of the self-energy functional approach, see M. Potthoff, Eur. Phys. J. B 32, 429 (2003).

[19] K. Inaba et al., Phys. Rev. B 72, 085112 (2005).

[20] C. Knecht, N. Blümer, and P. G. J. van Dongen, Phys. Rev. B 72, 081103(R) (2005).

[21] S. Biermann, L. de'Medici, and A. Georges, Phys. Rev. Lett. 95, 206401 (2005).

[22] A. Georges, G. Kotliar, W. Krauth, and M. J. Rozenberg, Rev. Mod. Phys. 68, 13 (1996); D. Vollhardt, Lecture Notes for the 9th Jerusalem Winter School for Theoretical Physics, Jerusalem, 30 December 1991-8 January 1992, edited by V. J. Emery (World Scientific, Singapore, 1993).

[23] K. G. Wilson, Rev. Mod. Phys. 47, 773 (1975); H. R. Krishna-murthy, J. W. Wilkins, and K. G. Wilson, Phys. Rev. B 21, 1003 (1980); for a recent review, including applications to lattice models, see: R. Bulla, T. Costi, and T. Pruschke, arXiv:cond-mat/0701105 [Rev. Mod. Phys. (to be published)].

[24] T. A. Costi, A. C. Hewson, and V. Zlatić, J. Phys. Condens. Matter 6, 2519 (1994).

[25] R. Bulla, Phys. Rev. Lett. 83, 136 (1999).

[26] W. Hofstetter, Phys. Rev. Lett. 85, 1508 (2000).

[27] N. Furukawa, J. Phys. Soc. Jpn. 63, 3214 (1994).

[28] A. Liebsch and T. A. Costi, Eur. Phys. J. B 51, 523 (2006).

[29] P. W. Anderson, J. Phys. C 3, 2436 (1970).

[30] T. A. Costi and A. Liebsch (to be published).

[31] For a comparison of QMC spectra, see A. Liebsch, arXiv:cond-mat/0610482. Note that, because of the $T=$ 0 quantum phase transition, the wide band at $T>0$ exhibits a smooth crossover rather than a sharp transition.

[32] A generalization of this model including a magnetic field has been discussed in: P. G. J. van Dongen and C. Leinung, Ann. Phys. (Leipzig) 509, 45 (1997).

[33] For a four-peaked structure in the spectral density of the one-band model within a finite cluster QMC calculation, see N. Tomita, M. Yamazaki, and K. Nasu, Phys. Rev. B 68, 035117 (2003). 